\title{
Survivable BBU Placement for C-RAN over Optical Aggregation Networks
}

\author{
Mohamed Shehata, Omran Ayoub, Francesco Musumeci, Massimo Tornatore \\ Department of Electronics, Information and Bioengineering, Politecnico di Milano, Italy \\ E-mails: \{mohamedkhaled.shehata; omran.ayoub; francesco.musumeci; massimo.tornatore\}@ polimi.it.
}

\begin{abstract}
Centralized Radio Access Network (C-RAN) is a novel mobile network architecture, designed to effectively support the challenging requirements of future $5 \mathrm{G}$ mobile networks. Unlike Distributed RAN (D-RAN), in CRAN, baseband units (BBUs) are located in a single physical location, called "BBU pool", connected to several Remote Radio Heads (RRHs) via the so-called "fronthaul" network. Following this centralization, designing a survivable C-RAN becomes crucial as BBU pool failures might cause service outage for large number of users. In this paper, we introduce the survivable BBU pool placement problem for C-RAN deployment over an optical aggregation network and formalize it by Integer Linear Programming (ILP). The ILP objective is to minimize the number of BBU pools, the number of used wavelengths and the computational resources. The results compare the aforementioned objectives and show the effect of the latency and of the transport-network capacity on the optimal solution.
\end{abstract}

Keywords: C-RAN, baseband unit, survivable, backup, failure.

\section{INTRODUCTION}

The design and operation of the upcoming $5 \mathrm{G}$ networks is challenged by the very stringent constraints of 5G in terms of tolerable latency and required data rate [1]. The main idea of C-RAN is that multiple BBUs are placed in a single physical location (BBU pool), which is connected to the RRHs through a high capacity "fronthaul" network. Thanks to centralization, the baseband resources in the BBU pool can be virtualized and shared among several Base Stations (BSs); hence, a significant reduction in the overall computational resources can be achieved due to multiplexing gain.

Despite the advantages provided by C-RAN, the fronthaul network must provide very high bandwidth with very low latency, leading to high transport-network cost. Optical aggregation networks based on wavelength division multiplexing (WDM) are considered a relevant candidate solution to meet the fronthaul requirements [2]. In this context, the question that arises is: where to place the BBU pool in the aggregation network? The placement problem aims to choose a BBU pool location, which meets the latency and bandwidth requirements while maximizing the aforementioned centralization benefits.

An important aspect in the placement problem is how to deal with BBU pool failures, as these failures might cause service outage for a large serving area with a significant number of users. This has recently motivated novel research on the design of survivable C-RAN. Survivable C-RAN has been rarely discussed in the literature. Ref. [3] defines a protection problem for cloud radio access network against BBUs and link failures. The authors present different approaches based on $1+1$ dedicated path protection and $1+1$ virtual machine replication through an ILP algorithm, but the authors have not considered delay and link capacity constraints. Ref. [4] proposes an algorithm to connect each RRH to two BBU pools, primary and backup, while reducing the number of the backup BBU ports among RRHs. Ref. [5] formulates an Integer Linear Program (ILP) problem named cost-resilience BBU selection, where a mobile network operator has to select BBU equipment from several cloud providers with different failure probability and cost. The objective function minimizes the BBU pool processing power and maximizes the resiliency. None of the previous works formulates an ILP problem for survivable BBU placement with considering latency and link capacity constraints. Moreover, we take into consideration the BBU pools dimension in terms of the computational resources.

In this paper, we investigate the survivable BBU placement problem for C-RAN deployment. When designing a resilient C-RAN, every RRH must be connected to two different BBU pools, one as a working (primary) pool and the second one as a backup pool. We formulate and solve the survivable BBU placement problem over an optical aggregation network using an Integer Linear Program (ILP). We consider an objective function with multiple optimization goals i) minimizing the number of BBU pools, ii) minimizing the number of used wavelengths and iii) minimizing the computational processing. We observe that latency and links capacity contributes to the optimal solution. Moreover, minimizing the computational resources results in additional savings compared to minimizing the number of pools.

The remainder of this paper is organized as follows. Section 2 discusses the considered network architecture. Section 3 presents our ILP problem formulation. Section 4 shows the illustrative numerical results. Section 5 concludes the work. 


\section{NETWORK ARCHITECTURE}

We consider an optical aggregation network where nodes are hierarchically-organized in a two-stages architecture composed by Access COs and Main COs as shown in Fig.1. For simplicity, we assume that every node contains a $\mathrm{RRH}$ and also represents a potential BBU pool. The main latency contributors considered in this work are the fiber propagation latency and the electronic switch latency. For the sake of simplicity, we assume Opaque optical network and that every RRH-BBU connection (the so-called "fronthaul" connection) is transported over a dedicated wavelength. To calculate the computational effort network (expressed in Giga Operations Per Second (GOPS)) required by the various BBUs and the associated multiplexing gain for different pool dimensions we use our model in [6].

\section{PROBLEM FORMULATION}

The survivable BBU pool placement problem is defined as follows: Given: network topology, number of wavelengths per link, maximum allowed fronthaul latency, and computational effort needed by a pool serving given number of cell sites.

Output: placement of BBUs such that each RRH is connected to two BBU pools one as a primary one and one as backup, and routing of traffic between the RRHs to the BBU pool.

Objective: minimizing i) Number of BBU pools, ii) Overall computational effort of all BBU pools and iii) the used number of fiber links.

\section{Input sets and parameters:}

a) $\quad N$ is set of nodes in the physical network, $i, j, m, n \in N$.

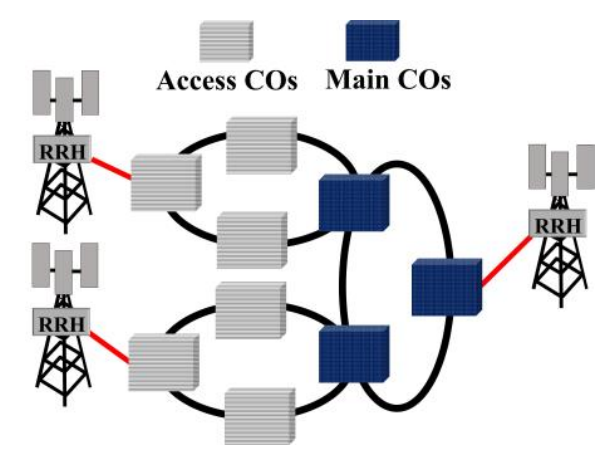

Figure 1. Network Architecture

b) $d_{i j}$ is the propagation delay introduced by the physical link $i-j$.

c) $D$ is the maximum allowable delay between cell site and the BBU pool fronthaul delay.

d) $W$ is the number of wavelengths per each physical link.

e) $C_{q}$ is the computational effort in GOPS needed by a pool if it serves q RRHs.

f) $C$ is the maximum computational effort in GOPS that can be accommodated by a pool.

II. Decision Variables:

a) $\quad k_{i}=1$, if node $i$ hosts a BBU pool (binary).

b) $x_{i, m}=1$, if cell site $m$ is assigned to a primary BBU pool at node $i$ (binary).

c) $z_{j, m}=1$, if cell site $m$ is assigned to a backup BBU pool at node $j$ as a backup pool (binary).

d) $y_{i j}^{m n}=1$, if virtual link $m-n$ to reach a primary pool is routed over the physical link $i-j$ (binary).

e) $t_{i j}^{m n}=1$, if virtual link $m-n$ to reach a backup pool is routed over the physical link $i-j$ (binary).

f) $\quad b_{i, q}=1$, if the BBU pool hosted by node $i$ serves $q$ RRHs as primary and backup (binary). If node $i$ does not host a pool, then $b_{i, 0}=1$.

\section{Objective function:}

The multi-objective function illustrated in Eq. (1) is composed of three parts. First term aims at minimizing the number of BBU pools. Second term minimizes the number of used wavelengths in the transport network. The last term minimizes the total computational effort required by the network.

$$
\min \left(\beta \sum_{i} k_{i}+\gamma \sum_{i j} \sum_{m n}\left(y_{i j}^{m n}+t_{i j}^{m n}\right)+\alpha \sum_{i} \sum_{q} b_{i, q} C_{q}\right)
$$

Parameters $\alpha, \beta$ and $\gamma \in[0,1]$ can be tuned to select the primary objective of the optimization.

\section{Constraints:}

$$
\begin{aligned}
& \sum_{i} x_{i, m}=1 \quad \forall m \quad(2) \quad, \quad \sum_{i} z_{j, m}=1 \quad \forall m \\
& x_{i, m}+z_{i, m} \leq 1 \quad \forall i, \forall m \quad \text { (4) } \quad, \quad k_{i} \geq \frac{\sum_{m}\left(x_{i, m}+z_{i, m}\right)}{M} \quad \forall m \\
& \sum_{q} b_{i, q} C_{q} \leq C \quad \forall i \quad(6) \quad, \quad \sum_{q} q b_{i, q}=\sum_{m}^{M} x_{i, m}+z_{i, m} \quad \forall i \\
& \sum_{q} b_{i, q}=1 \quad \forall i \quad(8) \quad, \quad \sum_{m n}\left(y_{i j}^{m n}+t_{i j}^{m n}\right) \leq W \quad \forall i j
\end{aligned}
$$




$$
\begin{aligned}
& \sum_{i j} y_{i j}^{m n} d_{i j} \leq D \quad \forall m n \quad(10) \quad, \quad \sum_{i j} t_{i j}^{m n} d_{i j} \leq D \\
& \sum_{j}\left(y_{i j}^{m n}-y_{j i}^{m n}\right) \\
& =\left\{\begin{array}{cl}
x_{n, i}, & i=m, m \neq n \\
-x_{i, m}, & i=n, m \neq n \\
0, & \text { otherwise }
\end{array}\right. \\
& \forall m n, \forall i \\
& \begin{array}{c}
\sum_{j}\left(t_{i j}^{m n}-t_{j i}^{m n}\right) \\
=\left\{\begin{array}{cl}
z_{n, i}, & i=m, m \neq n \\
-z_{i, m}, & i=n, m \neq n \\
0, & \text { otherwise }
\end{array} \quad \forall m n, \forall i\right.
\end{array}
\end{aligned}
$$

Eqns. (2) and (3) enforce that each RRH is associated with exactly one primary BBU pool and one backup BBU pool. Eq. (4) enforces primary BBU pool and backup BBU pool to be at different nodes for the same RRH. Eq. (5) is needed to identify BBU pools as the nodes which host at least one (primary or backup) BBU. Eq. (6) to guarantee that GOPS of all the BBUs aggregated in certain pool does not exceed the pool capacity. Eqns. (7) and (8) ensure that the number of RRHs served by pool $i$ equals the sum of RRHs assigned to that pool $i$. Eq. (9) guarantees that capacity of virtual links routed over a certain physical link did not exceed its capacity. Eqns. (10) and (11) ensure meeting the delay requirements. Eqns. (12) and (13) are the flow constraints, which guarantee that all virtual links connecting the RRHs with the primary BBU pools and backup BBU pools are mapped on a physical links.

\section{CASE STUDY AND RESULTS}

In this work, we consider a network with 20 nodes (4 main COs and 16 access COs) uniformly distributed over a dense urban square region of $5 \mathrm{~km}^{2}$. Nodes are organized in ring and spur physical topologies and are connected via fiber links as illustrated in Section 2. We consider a $20 \mu$ s latency for each electronic switch. To solve our optimizations we used ILOG CPLEX 12.0 on a workstation equipped with $8 \times 2 \mathrm{GHz}$ processors and $32 \mathrm{~GB}$ of RAM. In the following, we show the results for three different objective functions. 1) First objective function (O1): we optimize the number of BBU pools as a first objective, then the number of used wavelengths as the second objective. 2) Second objective function (O2): we first optimize the number of used wavelengths then the number of BBU pools. 3) Third objective function (O3): we optimize first the computational effort, then the number of BBU pools, then the number of used wavelengths. Numerical results reported below are obtained by averaging over several randomly-generated networks. Note that the number of wavelengths is counted as the summation of the variables $y_{i j}^{m n}$ and $t_{i j}^{m n}$ i.e., $\sum_{i j} \sum_{m n}\left(y_{i j}^{m n}+t_{i j}^{m n}\right)$.

Figure 2 shows the number of BBU pools and the number of used wavelengths (left and right bars, respectively) as a function of the maximum allowable fronthaul latency (D) considering objective function $\mathrm{O} 1$ and link capacity $W=6$. Note that, no solution could be obtained before $D=25 \mu \mathrm{s}$, as the strict latency does not allow BBUs to be hosted outside the cell site, hence the backup cannot be placed within acceptable latency. In general, as expected, for low latency values $(D<45 \mu s)$ all nodes must be activated as pools. In this case, the tight latency values force one BBU (the primary or the backup) to be placed at the cell site and the other BBU is placed in the adjacent node. As the latency increases the number of BBU pools decreases as expected. The minimum number of pools achieved is 4 , as link capacity does not allow to achieve complete centralization. In Fig. 3, we increase the link capacity $W$ to 20 resulting in reducing the minimum number of BBU pools to 2 . This confirms that link capacity was the main constraint for BBU consolidation at high latency in Fig. 2 . In both cases $(W=6$ and $W=$ 20 ) as the number of BBU pools decreases, the number of required wavelengths increases. In fact, for high number of pools ( $D<45 \mu \mathrm{s}$ ) one BBU (primary or backup) is placed at the cell site, and in this case no transport link is occupied by this BBU. If we relax the latency constraint $(D \geq 45 \mu s)$ BBUs (primary and backup) can be consolidated in central pools, requiring more RRH-BBU connections, so more wavelengths, to be used.

Figure 4 shows the number of BBU and wavelengths as a function of the maximum allowable fronthaul latency (D) considering objective function $\mathrm{O} 2$ and $W=6$. In this case, the number of BBU pools and number of used wavelengths are constant over the different latencies values. As the first objective of $\mathrm{O} 2$ is to minimize the number of wavelengths, the solver keeps one BBU (the primary or the backup) at the cell site and allocates the other BBU in the adjacent node. Minimizing the overall number of wavelengths, does not give the priority for the minimizing the primary wavelengths rather than the backup ones. For example, in low latency value $(D \leq 45 \mu s)$ most of the backup BBUs are placed at the cell site and the primary placed at the adjacent nodes. This leads to higher number of primary wavelengths than the backup ones but the total number of wavelengths is minimized. 


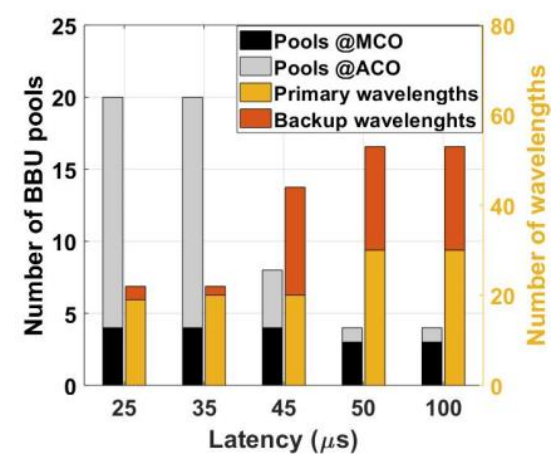

Figure 2. Number of $B B U$ pools and wavelengths (objective $=01, W=6$ )

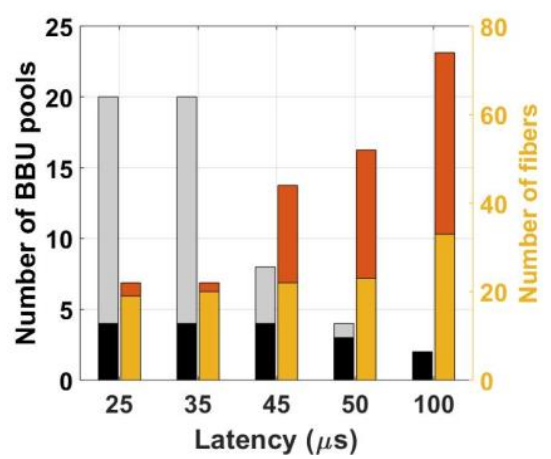

Figure 3. Number of $B B \cup$ pools and wavelengths (objective $=01, W=20$ )

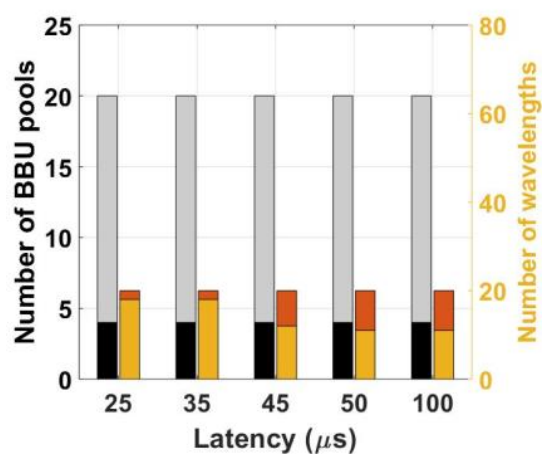

Figure 4. Number of $B B \cup$ pools and wavelengths (objective $=02, W=6$ )

Figures 5 and 6 compare objectives $\mathrm{O} 1$ and $\mathrm{O} 3$ in terms of the number of pools and the amount of Computational Effort (CE). We consider $D=100 \mu s, W=20$ and define $n$ as the maximum number of aggregated BBUs per pool. Figure 5 shows the number of BBU pools for $\mathrm{O} 1$ and $\mathrm{O} 3$ considering different $n$ values. The results show that adding a term to minimize the computational effort does not contribute to the number of the BBU pools as no difference is observed between $\mathrm{O} 1$ and O3. Figure 6 shows the computational effort in GOPS corresponding to each objective function and $n$. Despite $\mathrm{O} 1$ and $\mathrm{O} 3$ have the same number of BBU pools, the two objectives provide different amount of CE. While minimizing the GOPS in O3 not only guarantee the minimum number of pools but also choose combinations for the number of BBUs to be placed in the pools with higher multiplexing gain.

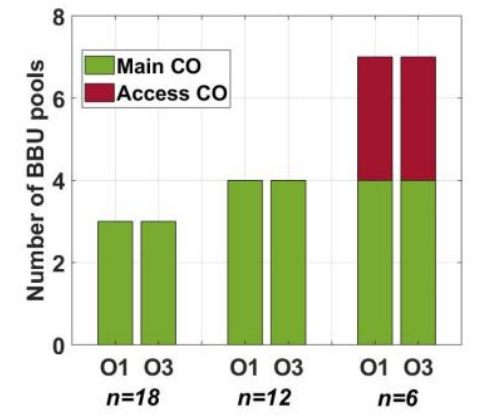

Figure 5. Number of $\mathrm{BBU}$ pools for $\mathrm{O} 1$ and $\mathrm{O} 3$

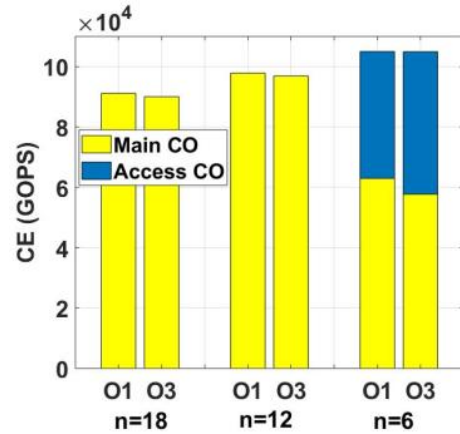

Figure 6. Computational effort in GOPs for 01 and $\mathrm{O3}$

\section{CONCLUSION}

In this work, we have investigated the survivable C-RAN deployment problem over an optical aggregation network. We have formulated the problem through an ILP model with different objectives, minimizing number of BBU pools, number of used wavelengths and overall computational effort. We have then observed the impact of latency and link capacity on the optimal solution. Finally, we show that minimizing the computational effort results in a slight additional savings compared to the traditional minimization of the number of BBU pools. As a future work, we will consider link protection as well as grooming and wavelength continuity constraints. Moreover, we are developing a heuristic algorithm to deal with large network dimensions.

\section{ACKNOWLEDGEMENTS}

The research leading to these results has received funding from the European Community under grant agreement no. 761727 Metro-Haul project.

\section{REFERENCES}

[ 1 ] E. Hossain and M. Hasan, "5G cellular: key enabling technologies and research challenges," IEEE Instrumentation \& Measurement Magazine, vol. 18, no. 3, pp. 11-21, 2015.

[2] Musumeci, Francesco, et al. "Optimal BBU placement for 5G C-RAN deployment over WDM aggregation networks," IEEE/OSA Journal of Lightwave Technology 34.8 (2016): 1963-1970

[ 3 ] Colman-Meixner, Carlos, et al. "Resilient cloud network mapping with virtualized BBU placement for cloud-RAN." IEEE conference on Advanced Networks and Telecommunications Systems (ANTS), India, November 2016.

[ 4 ] Khorsandi, Bahare Masood, et al. "Survivable BBU Hotel placement in a C-RAN with an Optical WDM Transport." -13th International Conference Design of Reliable Communication Networks ( DRCN), Germany, March 2017

[ 5 ] Lyazidi, Mohammed Yazid, et al. "A Novel Optimization Framework for C-RAN BBU Selection based on Resiliency and Price." IEEE 86th Vehicular Technology Conference (VTC), Canada, September 2017.

[ 6 ] Shehata, Mohamed, et al. "C-RAN baseband pooling: Cost model and multiplexing gain analysis." 19th International Conference on Transparent Optical Networks (ICTON), Spain, July 2017. 